\title{
Association of BoLA-DRB3.2 Alleles with Resistance and Susceptibility to Persistent Lymphocytosis in BLV Infected Cattle in Argentina
}

\author{
${ }^{1,2}$ C.J. Panei, ${ }^{1}$ K. Suzuki, ${ }^{1,3}$ M.G. Echeverría, ${ }^{1,4}$ M.S. Serena, \\ ${ }^{1,4} \mathrm{G}$.E. Metz and ${ }^{1}$ E.T. González \\ ${ }^{1}$ Cátedra de Virología, Facultad de Ciencias Veterinarias, \\ Universidad Nacional de La Plata (FCV-UNLP), La Plata, 1900, Argentina \\ ${ }^{2}$ Comisión de Investigaciones Científicas de la Provincia de Buenos Aires (CIC), \\ La Plata, 1900, Argentina \\ ${ }^{3}$ Instituto de Genética Veterinaria Fernando Noel Dulout, \\ Facultad de Ciencias Veterinarias, Centro Científico Técnico de La Plata, \\ Consejo Nacional de Investigaciones Científicas y Técnicas \\ (IGEVET-FCV-CCT La Plata CONICET), La Plata, 1900, Argentina \\ ${ }^{4}$ CONICET, Buenos Aires, 1033, Argentina
}

\begin{abstract}
The objective of this preliminary study was to describe alleles of bovine major histocompatibility complex class II BoLA-DRB3.2 gene associated with resistance and susceptibility to Persistent Lymphocytosis (PL) in Bovine leukaemia virus (BLV) infected cattle, to set a direction for the future studies. A total of 81 dairy cattle in Argentina were investigated for the distribution of BoLA-DRB3.2 alleles. The study cattle were categorized into 3 groups as follows: Category $\mathrm{PL}^{+}$(BLV seropositive with PL); Category $\mathrm{PL}^{-}$(BLV seropositive without $\mathrm{PL}$ ) and Category $\mathrm{BLV}^{-}$(BLV seronegative). The alleles were identified by polymerase chain reaction with subsequent analysis of restriction fragment length polymorphism. The association between the BoLA-DRB3.2 alleles and the $\mathrm{PL}^{+}$and $\mathrm{PL}^{-}$categories was determined as the odds ratio. Some alleles BoLADRB3.2 mediating (1) resistance and (2) susceptibility to PL were observed, respectively. A stochastic model including Shannon's diversity index calculation was run based on Markov-Chain Monte Carlo simulation method. The Shannon's diversity indices in each of the cattle categories indicated that the richness of gene diversity decreased as leading to a clinical stage of BLV. This relates to the existence of alleles mediating resistance and susceptibility to PL. The stochastic prediction with inclusion of uncertainty is important which can serve as a future study beacon.
\end{abstract}

Key words: Holando-Argentino, MHC, modelling, PCR-RFLP, South America

\section{INTRODUCTION}

Genes from the Major Histocompatibility Complex (MHC) are exceptionally appealing because they are associated with genetic resistance and susceptibility to a broad range of diseases (Sharif et al., 1998; Rupp et al., 2007). Earlier studies showed that some bovine

Corresponding Author: K. Suzuki, PROVETSUR, Facultad de Ciencias Veterinarias, Universidad Nacional de La Plata, CC296, La Plata, B1900AVW, Argentina Tel/Fax: +542214253276 
MHC class II BoLA-DRB3.2 gene polymorphisms were correlated with resistance and susceptibility to the development of Persistent Lymphocytosis (PL) caused by Bovine leukaemia virus (BLV) infection (Xu et al., 1993, Zanotti et al., 1996). The range of BoLA-DRB3.2 alleles associated with resistance (DRB3.2* $11,{ }^{*} 23$ and ${ }^{*} 28$ ) and susceptibility (DRB3.2*8, ${ }^{*} 16, * 22$ and $* 24$ ) to PL in Holstein Friesian cattle was confirmed (Xu et al., 1993; Lewin et al., 1999). It is believed that the allelic differences have an impact on the binding and orientation of viral peptides, consequently, determining allele-specificity differences in the spectrum of peptides present in the immune system. These differences have important results for infection resistance and susceptibility (Tiwari and Terasaki, 1985; Zavala-Ruiz et al., 2004). Evidence was showed that BLV-infected cattle chose for the presence of the DRB3.2* 11 allele carry fewer infected lymphocytes than other infected cattle (Mirsky et at., 1998). These findings need to be interpreted carefully, as the sampling methods used were not optimal for the intended purpose or might not be correct statistically. The objective of this preliminary study was to describe BoLA-DRB3.2 alleles associated with resistance and susceptibility to PL in BLV infected dairy cattle using stochastic prediction with inclusion of uncertainty, to set a direction for the future studies.

\section{MATERIALS AND METHODS}

This study was conducted between December 2007 and March 2009. Samples from a total of 81 Holando-Argentino dairy cattle between 2 and 6 years old reared at our Faculty ranch in Santa Catalina, Buenos Aires Province, Argentina were examined for the distribution of BoLA-DRB3.2 alleles. Agar gel immunodiffusion and indirect enzyme-linked immunosorbent assay were used to determine BLV seropositivity (González et al., 2007). PL was defined by a property of lymphocyte counts of more than $10^{4} \mu \mathrm{L}^{-1}$ in peripheral blood (Tolle, 1965). According to these serological and haematological methods, the study cattle were categorized into 3 groups as follows: Category $\mathrm{PL}^{+}$(BLV seropositive with $\mathrm{PL}, \mathrm{N}=42)$; Category $\mathrm{PL}^{-}(\mathrm{BLV}$ seropositive without $\mathrm{PL}, \mathrm{N}=25$ ) and Category $\mathrm{BLV}^{-}$(BLV seronegative, $\mathrm{N}=14$ ). The BoLA-DRB3.2 alleles were identified by polymerase chain reaction with subsequent analysis of restriction fragment length polymorphism (PCR-RFLP) (Van-Eijk et al., 1992). Allele frequencies for each of the cattle categories and both observed and expected frequencies of heterozygotes of the total study cattle from the Hardy-Weinberg equilibrium were obtained using Arlequin software version 3.11 (http://cmpg.unibe.ch/software/arlequin3/). The association between the BoLA-DRB3.2 alleles and the $\mathrm{PL}^{+}$and $\mathrm{PL}^{-}$categories was determined as the Odds Ratio (OR) (Dohoo et al., 2003). If any value in a $2 \times 2$ table was zero, Haldane's modified Woolf formula for OR was used (Tiwari and Terasaki, 1985). An OR value $>1$ indicated that those animals carrying the allele were at lower risk of developing the PL and were considered as resistant. An OR value $<1$ indicated that those carrying the allele were at higher risk of developing the PL and were considered as susceptible. Then, the Fisher's exact test was performed to investigate statistical significance of the OR (Dohoo et al., 2003).

The proportion of individual alleles in a cattle category that belong to different allele was used to compute gene diversity indices [Shannon's diversity index $(\mathrm{H})$ ] (Begon et al., 2006):

$$
\mathrm{H}=-\sum_{\mathrm{i}=1}^{\mathrm{S}} \mathrm{P}_{\mathrm{i}} \ln \left(\mathrm{P}_{\mathrm{i}}\right)
$$


where, $S$ is the number of alleles in a cattle category and $P_{i}$ is the proportion of individual alleles in the cattle category that belong to allele $i$. The $H$ value increases with richness of gene diversity in a cattle category.

This corresponds to the hypothesis that those animals carrying alleles resistant to PL remain at lower clinical stage (Category $\mathrm{PL}^{-}$) and only those carrying alleles susceptible to PL can enter higher clinical stage (Category $\mathrm{PL}^{+}$). The proportion data describing the distribution of individual alleles in each of the cattle categories were also used to estimate the chances or specified probabilities, which reflect the frequencies of the alleles in the cattle categories. The multinomial distribution was adopted for modelling the data and the Dirichlet distribution was used as prior distribution (Congdon, 2007). This stochastic model including Shannon's diversity index calculation was run based on Markov-Chain Monte Carlo simulation method in the WinBUGS software version 1.4.3 (www.mrc-bsu. cam.ac.uk/bugs/).

\section{RESULTS AND DISCUSSION}

We identified 17 BoLA-DRB3.2 alleles defined according to the PCR-RFLP nomenclature and represented the distribution of the allele frequencies. The most frequent alleles were the BoLADRB3.2* $10, * 8, * 24$ and ${ }^{*} 15$ alleles, with frequencies ranging between 10 and $21 \%$. The other 13 alleles had frequencies less than 10\% (Table 1). Observed and expected heterozygosities for all the study cattle were 40 and 89 , respectively ( $N=81 ; p<0.001)$. Alleles BoLA-DRB3.2*11, *23,*25, *28 and *40 mediating resistance to PL were observed. Alleles BoLA-DRB3.2*22 and *24 mediating susceptibility to PL were also observed (Table 1).

The result of taking 100,000 samples from the posterior distribution provided the predicted frequencies of BoLA-DRB3.2 alleles as well as Shannon's diversity indices in each of the cattle categories (Fig. 1a-c). The Shannon's diversity indices in each of the cattle categories $\left[\mathrm{BLV}^{-}(2.41) \mathrm{B} \rightarrow \mathrm{PL}^{-}(2.38) \mathrm{B} \rightarrow \mathrm{PL}^{+}(2.32)\right]$ indicated that the richness of gene diversity or the number of alleles decreases as leading to a clinical stage.

The results that alleles BoLA-DRB $3.2^{*} 11,{ }^{*} 23$ and $* 28$ mediating resistance to PL and alleles BoLA-DRB3.2*22 and *24 mediating susceptibility to PL were in agreement with the

Table 1: Frequencies of BoLA-DRB3.2 alleles based on PCR-RFLP method nomenclature in the cattle category

\begin{tabular}{|c|c|c|c|c|c|c|c|c|}
\hline \multirow[b]{2}{*}{ Alleles } & \multicolumn{4}{|c|}{ Cattle category (No. of alleles) } & \multirow{2}{*}{$\begin{array}{l}\text { Odds } \\
\text { ratio }^{1}\end{array}$} & \multicolumn{2}{|l|}{$95 \% \mathrm{CI}$} & \multirow{2}{*}{$\begin{array}{l}\text { Fisher's } \\
\text { exact P }\end{array}$} \\
\hline & $\mathrm{PL}^{+}(84)$ & $\mathrm{PL}^{-}(50)$ & $\mathrm{BLV}^{-}(28)$ & Total (162) & & Lower & Upper & \\
\hline$*^{* 1}$ & 0.01 & - & & 0.01 & 0.55 & 0.057 & 5.40 & 1.00 \\
\hline$* 8$ & 0.14 & 0.16 & 0.11 & 0.14 & 1.10 & 0.430 & 3.00 & 0.81 \\
\hline$* 10$ & 0.19 & 0.30 & 0.11 & 0.21 & 1.80 & 0.810 & 4.10 & 0.20 \\
\hline$* 11$ & - & 0.06 & 0.07 & 0.03 & 12.00 & 1.500 & 100.00 & $<0.05$ \\
\hline$* 15$ & 0.13 & 0.08 & 0.04 & 0.10 & 0.58 & 0.170 & 1.90 & 0.41 \\
\hline$* 16$ & 0.10 & 0.02 & 0.18 & 0.09 & 0.19 & 0.024 & 1.60 & 0.15 \\
\hline$* 20$ & 0.08 & 0.04 & - & 0.06 & 0.46 & 0.091 & 2.30 & 0.48 \\
\hline$* 22$ & 0.12 & - & - & 0.06 & 0.07 & 0.010 & 0.53 & $<0.05$ \\
\hline$* 23$ & - & 0.08 & 0.14 & 0.05 & 16.00 & 2.000 & 130.00 & $<0.05$ \\
\hline$*_{24}$ & 0.17 & - & 0.24 & 0.13 & 0.048 & 0.010 & 0.36 & $<0.05$ \\
\hline$* 25$ & - & 0.1 & - & 0.03 & 20.00 & 2.600 & 160.00 & $<0.05$ \\
\hline "26 & 0.02 & 0.04 & - & 0.02 & 1.70 & 0.230 & 13.00 & 0.62 \\
\hline$* 28$ & - & 0.04 & - & 0.01 & 8.70 & 1.000 & 76.00 & $<0.05$ \\
\hline$* 36$ & 0.04 & - & - & 0.02 & 0.23 & 0.028 & 1.90 & 0.27 \\
\hline$* 40$ & - & 0.06 & 0.04 & 0.02 & 12.00 & 1.500 & 100.00 & $<0.05$ \\
\hline "41 & - & - & 0.07 & 0.01 & - & - & - & - \\
\hline$* 51$ & - & 0.02 & - & 0.01 & 3.40 & 0.310 & 38.00 & 0.56 \\
\hline
\end{tabular}

${ }^{1}$ The association between the BoLA-DRB3.2 alleles and the $\mathrm{PL}^{+}$and $\mathrm{PL}^{-}$categories was determined. A number $<1$ shows an association with $\mathrm{PL}^{+}$and number $>1$ with $\mathrm{PL}^{-}$. BLV seropositive with persistent lymphocytosis ( $\left.\mathrm{PL}^{+}\right)$, seropositive without persistent lymphocytosis $\left(\mathrm{PL}^{-}\right)$and seronegative $\left(\mathrm{BLV}^{-}\right)$ 


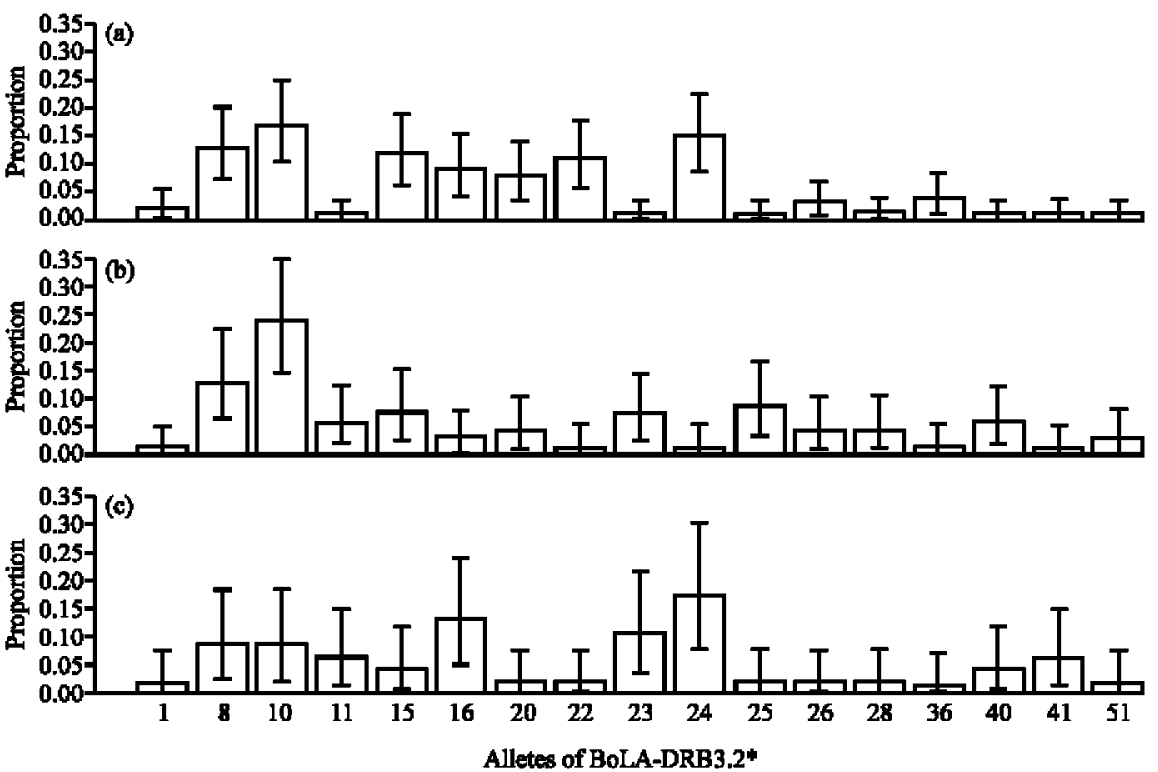

Fig. 1: Predicted frequencies of BoLA-DRB3.2 alleles in the cattle category [(a) BLV seropositive with persistent lymphocytosis $\left(\mathrm{PL}^{+}[\mathrm{n}=84 ; \mathrm{H}=2.32(95 \% \mathrm{BCI}\right.$ : 2.18-2.45)], (b) seropositive without persistent lymphocytosis ( $\mathrm{PL}^{-}[\mathrm{n}=50 ; \mathrm{H}=2.38$ (95\% BCI: 2.19-2.55)] and (c) seronegative (BLV- $[\mathrm{n}=28 ; \mathrm{H}=2.41(95 \%$ BCI: 2.20-2.58)]. The columns and error bars represent the means of the posterior distribution and $95 \%$ Bayesian credible intervals (BCIs), respectively. Estimates of gene diversity in each of the categories are also shown as Shannon's diversity index (H) and $95 \%$ BCls. The $\mathrm{H}$ value lowers with poorness of gene diversity in a cattle category

previous findings (Xu et al., 1993; Lewin et al., 1999). Alleles BoLA-DRB3.2*25 and *40 also showed significant association for resistance to PL. These results, as far as we know, have not been observed by previous studies. Particularly the allele BoLA-DRB3. $2^{*} 25$ had the highest $\mathrm{OR}$ value indicating strong association with $\mathrm{PL}^{-}$. Udina et al. (2003) reported that the allele BoLA-DRB3.2*25 in Black Pied cattle breed in Russia possessed neutral properties in relation to PL and in the absence of other reports from the world, these results were therefore considered not to be generalized. These results in conjunction with the significantly small value of observed heterozygosities (or deviations from Hardy-Weinberg equilibrium) could be partly explained by inbreeding as well as assortative mating in the study population.

The decrease of the richness of gene diversity as leading to a clinical stage was observed. This relates to the existence of alleles mediating resistance and susceptibility to PL. Alleles BoLA-DRB3.2 mediating (1) resistance and (2) susceptibility to PL were predominant in the categories (1) $\mathrm{PL}^{-}$and (2) $\mathrm{PL}^{+}$, respectively. This shows that Shannon's diversity index, originally from measuring ecological diversity, would be a beneficial indicator at cattle population level in combination with the $O R$ at individual allele level.

The limitation of this preliminary study is that the sample size was not relatively large $(\mathrm{N}=81$ ) which might cause a sampling effect and these findings may not apply to all dairy 
cattle in Buenos Aires Province. However, we will never know the proportions of individual alleles in the cattle categories precisely, even if millions of animals are investigated. Therefore we conclude that this stochastic prediction with inclusion of uncertainty (Fig. 1) is advantageous and considerably important in population genetics which can serve as a future study beacon. Further investigations into the comparison of this stochastic prediction with frequencies of BoLA-DRB3.2 alleles in the cattle category, when the number of study animals as well as study location is increased, would be of interest.

\section{ACKNOWLEDGMENTS}

This study was financially supported in part by Secretaría de Ciencia y Técnica de Universidad Nacional de La Plata. We appreciate the FCV-UNLP and CIC's support to implement this study. We also thank the Japan International Cooperation Agency for helping us through the project, commonly known as Proyecto de desarrollo profesional continuo para los veterinarios del Sur (PROVETSUR).

\section{REFERENCES}

Begon, M., C.R. Townsend and J.L. Harper, 2006. Ecology: From Individuals to Ecosystems. 4th Edn, Blackwell Publishing Ltd, Oxford, ISBN: 978-1405111171.

Congdon, P., 2007. Bayesian Statistical Modelling. 2nd Edn., John Wiley and Sons, Oxford, ISBN: 978-0470018750.

Dohoo, I., W. Martin and H. Stryhn, 2003. Veterinary Epidemiologic Research. 1st Edn., AVC, Charlottetown, ISBN: 0-919013-41-4.

González, T., M. Licursi and E. Bonzo, 2007. Enzootic bovine leukosis: Performance of an indirect ELISA applied in serological diagnosis. Braz. J. Microbiol., 38: 1-5.

Lewin, H.A., G.C. Russell and E.J. Glass, 1999. Comparative organization and function of the major histocompatibility complex of domesticated cattle. Immunol. Rev., 167: 145-158.

Mirsky, M.L., C. Olmstead, Y. Da and H.A. Lewin, 1998. Reduced Bovine leukaemia virus proviral load in genetically resistant cattle. Anim. Genet., 29: 245-252.

Rupp, R., A. Hernandez and B.A. Mallard, 2007. Association of bovine leukocyte antigen (BoLA) DRB 3.2 with immune response, mastitis and production and type traits in Canadian Holsteins. J. Dairy Sci., 90: 1029-1038.

Sharif, S., B.A. Mallard, B.N. Wilkie, J.M. Sargeant and H.M. Scott et al., 1998. Associations of the bovine major histocompatibility complex DRB3 (BoLA-DRB3) alleles with occurrence of disease and milk somatic cell score in Canadian dairy cattle. Anim. Genet., 29: $157-160$.

Tiwari, J.L. and P.I. Terasaki, 1985. HLA and Disease Associations. 1st Edn., Springer-Verlag, New York, ISBN: 978-3540960812.

Tolle, A., 1965. On the determination of quantitative hematologic findings in the diagnosis of leukemia in cattle. Zentralbl Veterinarmed B, 12: 281-290 (In German).

Udina, I.G., E.E. Karamysheva, S.O. Turkova, A.R. Orlova and G.E. Sulimova, 2003. Genetic mechanisms of resistance and susceptibility to leukemia in Ayrshire and black pied cattle breeds determined by allelic distribution of gene Bola-DRB3. Russ. J. Genet, 39: 383-396.

Van-Eijk, M.J.T., J.A. Stewart-Haynes and H.A. Lewin, 1992. Extensive polymorphism of the BoLA-DRB3 gene distinguished by PCR-RFLP. Anim. Genet, 23: 483-496. 
Xu, A.L., M.J.T. Vaneijk, C. Park and H.A. Lewin, 1993. Polymorphism in BoLA-DRB3 exon 2 correlates with resistance to persistent lymphocytosis caused by Bovine leukemia virus. J. Immunol, 151: 6977-6985.

Zanotti, M., G. Poli, W. Ponti, M. Polli, M. Rocchi et al., 1996. Association of BoLA class II haplotypes with subclinical progression of Bovine leukaemia virus infection in Holstein-Friesian cattle. Anim. Genet, 27: 337-341.

Zavala-Ruiz, Z., I. Strug, B.D. Walker, P.J. Norris and L.J. Stern, 2004. A hairpin turn in a class II MHC-bound peptide orients residues outside the binding groove for $\mathrm{T}$ cell recognition. Proc. Natl. Acad. Sci. USA., 101: 13279-13284. 